\title{
AVALIAÇÃO DA CONDUTIVIDADE HIDRÁULICA EM ÁREAS DE RECARGA DO AQUÍFERO GUARANI-MICROBACIA HIDROGRÁFICA DO CÓRREGO ESPRAIADO (SP)
}

\begin{abstract}
A condutividade hidráulica do solo saturado (Ksat) constitui parâmetro de grande relevância nos estudos de vulnerabilidade natural e, por consequência, na avaliação de riscos ambientais. Quando se trata de áreas frágeis, como são as áreas de recarga de aquíferos sedimentares, a avaliação da condutividade hidráulica torna-se ainda mais importante. Para esse estudo, selecionou-se uma área de recarga direta do Aquífero Guarani, caracterizada pela microbacia do córrego Espraiado, localizada na região de Ribeirão Preto (SP). Os principais solos dessa microbacia são Latossolo Vermelho Distrófico psamítico (LVdq) e Neossolo Quartzarênico Órtico (RQo). Para a avaliação da condutividade hidráulica (Ksat) desses solos utilizouse o método da coluna saturada, cujos valores foram correlacionados com os de textura e estrutura do solo, considerados os parâmetros diretamente relacionados à Ksat. As correlações foram diretas entre os valores de Ksat e os de textura arenosa (areia). Já para a estrutura do solo, essa correlação ocorreu de forma indireta, indicando menor influência desse parâmetro sobre a condutividade hidráulica do solo saturado.
\end{abstract}

PALAVRAS-CHAVE: CONTAMINAÇÃO DE ÁGUA; VULNERABILIDADE NATURAL; ÁREAS FRÁGEIS; QUALIDADE AMBIENTAL.

* Geólogo, Doutor em Solos, Pesquisador da Embrapa Meio Ambiente, Jaguariúna, SP (e-mail: gomes@cnpma.embrapa.br).

** Engenheiro Agrônomo, Doutor em Planejamento Rural Sustentável, Pesquisador da Embrapa Meio Ambiente, Jaguariúna, SP (e-mail: lauro@cnpma.embrapa.br). 


\section{INTRODUÇÃO}

Nos estudos ambientais que envolvem a avaliação de risco torna-se imprescindível a caracterização da área exposta à carga potencialmente contaminante. Tal caracterização que envolve, entre outros aspectos, a identificação de sua vulnerabilidade natural, requer o conhecimento de alguns parâmetros físico-químicos e biológicos relativos aos compartimentos geológico e pedológico.

Em áreas de recarga de aquíferos sedimentares, a exemplo do Guarani, a vulnerabilidade natural foi avaliada como sendo de nível alto (SÃO PAULO, 1997; GOMES, SPADOTTO e PESSOA, 2002) pelas condições de favorecimento à infiltração e percolação da água no perfil do solo. A taxa de condutividade hidráulica do solo saturado (Ksat) está entre essas condições e tem se mostrado relativamente elevada para os principais solos dessas áreas, tendo por influência direta a sua textura e também sua estrutura.

A condutividade hidráulica expressa a drenabilidade natural do solo e, consequentemente, está relacionada com o transporte de água, soluto e substâncias químicas. O seu conhecimento é fundamental para a produção agrícola e para o manejo e conservação do solo e da água, bem como para a previsão de eventuais riscos de contaminação. Trata-se de parâmetro que depende de vários fatores (físicos, químicos e biológicos), dentre os quais se encontram a textura e a estrutura do solo.

Os solos com classes texturais diferentes podem evidenciar distribuição contrastante na sua porosidade e, por conseguinte, na Ksat. Segundo BRADY (1983), qualquer alteração no tamanho e configuração dos poros do solo resulta em mudanças também na condutividade hidráulica, sendo os macroporos responsáveis pela maior parte da movimentação saturada da água no solo.

Neste trabalho foi realizada a avaliação específica dos valores de condutividade hidráulica em solo saturado, considerando o Latossolo Vermelho Distrófico psamítico (LVdq) e o Neossolos Quartzarênico Órtico (RQo) de acordo com EMBRAPA (1999), obtidos em cinco (05) períodos distintos (1996, 1998, 2000, 2001 e 2003) em locais específicos da microbacia do Córrego Espraiado, região de Ribeirão Preto (SP) (EMBRAPA, 2004). Tal avaliação procurou dar ênfase à condutividade hidráulica saturada como parâmetro de referência e relevância nos estudos de vulnerabilidade natural de áreas frágeis, como são as de recarga direta do Aquífero Guarani.

\section{MATERIAL E MÉTODOS}

A condutividade hidráulica foi avaliada para as condições de solo saturado (Ksat), conforme o método adotado por KRAMER (1969), FERNANDES et al. (1983) e YOUNGS (1991), a partir de um sistema ajustável de permeâmetros com lâmina d'água constante igual a $2 \mathrm{~cm}$. As amostras de solo foram submetidas a pré-umedecimento por capilaridade, durante 24 horas, com o propósito de eliminar o ar existente nos seus espaços vazios. Após esse período, foram feitas leituras durante 7 horas consecutivas. Avaliou-se a condutividade hidráulica utilizando-se a equação de Darcy:

$$
\text { Ksat }=\text { Q.At }{ }^{-1} \times \text { L.H }^{-1}
$$

Em que:

Ksat = condutividade hidráulica saturada $\left(\mathrm{cm} \cdot \mathrm{h}^{-1}\right)$; 
$\mathrm{Q}=$ volume de água percolada no tempo $\left(\mathrm{cm}^{3}\right)$;

$\mathrm{A}=$ área de secção transversal do tubo $\left(\mathrm{cm}^{2}\right)$;

$\mathrm{H}=$ carga hidráulica total $(\mathrm{cm})$;

$\mathrm{L}=$ comprimento da coluna de material de solo $(\mathrm{cm})$.

Os dados de textura foram obtidos pelo método da pipeta com o uso de $\mathrm{NaOH}$ 0,5 mol/L e agitação mecânica para obtenção da dispersão dos coloides (EMBRAPA, 1979). As frações granulométricas foram classificadas de acordo com as normas da Sociedade Brasileira de Ciência do Solo (SANTOS et al., 2005).

Obteve-se a estrutura pelo método indireto (tamisamento úmido), conforme Yooder (1936), citado por KIEHL (1979), e LACERDA et al. (2005), com adoção das classes de diâmetro de agregados >2 mm; 2 -1 mm; 1 - 0,5 mm; 0,5-0,25 mm e <0,25 mm.

\section{RESULTADOS E DISCUSSÃO}

Analisando as Tabelas 1 e 2, referentes à granulometria, verifica-se que o Latossolo Vermelho Distrófico psamítico (LVdq) apresentou média variando de 71 a 76\% de fração areia (grossa + fina). Já o Neossolo Quartzarênico Órtico (RQo) revelou média bem mais elevada, 87 a 92\%. Tais solos são muito arenosos e suas texturas possibilitam elevada capacidade de percolação, sobretudo sob condições de solo saturado, contribuindo para o incremento da condutividade hidráulica.

\section{TABELA 1 - RESULTADOS DE ANÁLISE GRANULOMÉTRICA DO LATOSSOLO VERMELHO DISTRÓFICO PSAMÍTICO (LVDQ) DA MICROBACIA DO CÓRREGO DO ESPRAIADO, CONSIDERANDO A MÉDIA DE QUATRO PONTOS DE AMOSTRAGEM}

\begin{tabular}{|c|c|c|c|c|c|}
\hline \multirow{3}{*}{ Solo } & \multirow{3}{*}{$\begin{array}{l}\text { Profundidade } \\
\text { (cm) }\end{array}$} & \multicolumn{4}{|c|}{ Granulometria } \\
\hline & & Areia Grossa & Areia Fina & Silte & Argila \\
\hline & & \multicolumn{4}{|c|}{$(\%)$} \\
\hline \multirow{6}{*}{ LVdq } & $0-20$ & 30 & 44 & 7 & 19 \\
\hline & $20-40$ & 29 & 46 & 8 & 17 \\
\hline & $40-60$ & 23 & 48 & 7 & 22 \\
\hline & $60-80$ & 25 & 51 & 6 & 18 \\
\hline & $80-100$ & 32 & 42 & 7 & 19 \\
\hline & $100-120$ & 30 & 46 & 8 & 16 \\
\hline
\end{tabular}




\section{TABELA 2 - RESULTADOS DE ANÁLISE GRANULOMÉTRICA DO NEOSSOLO QUARTZARÊNICO ÓRTICO (RQo) DA MICROBACIA DO CÓRREGO DO ESPRAIADO, CONSIDERANDO A MÉDIA DE QUATRO PONTOS DE AMOSTRAGEM}

\begin{tabular}{|c|c|c|c|c|c|}
\hline \multirow{3}{*}{ Solo } & \multirow{3}{*}{$\begin{array}{l}\text { Profundidade } \\
(\mathrm{cm})\end{array}$} & \multicolumn{4}{|c|}{ Granul ometria } \\
\hline & & Areia Grossa & Areia Fina & Silte & Argila \\
\hline & & \multicolumn{4}{|c|}{$(\%)$} \\
\hline \multirow{6}{*}{ RQO } & $0-20$ & 40 & 51 & 4 & 5 \\
\hline & $20-40$ & 42 & 49 & 2 & 7 \\
\hline & $40-60$ & 39 & 48 & 4 & 9 \\
\hline & $60-80$ & 39 & 52 & 3 & 6 \\
\hline & $80-100$ & 42 & 50 & 2 & 6 \\
\hline & $100-120$ & 39 & 50 & 4 & 7 \\
\hline
\end{tabular}

Efetuou-se a análise de agregação para os dois tipos de solos visando complementar as informações relativas à condutividade hidráulica. Dentre as cinco classes de agregados consideradas, os resultados mostraram-se bem distintos entre os dois tipos de solos. No Latossolo Vermelho Distrófico psamítico (LVdq), o tamanho de agregado predominante correspondeu à classe de diâmetro entre 2-1 mm, constituindo cerca de $40 \%$ do total dos agregados (Tabela 3 e Figura 1). No Neossolo Quartzarênico Órtico (RQo), a classe predominante foi a de diâmetro entre 0,5-0,25 mm correspondendo a $65 \%$ dos agregados (Tabela 4 e Figura 1). Os coeficientes de variação foram muito baixos (5,5\% e 3,1\%, respectivamente), mas considerados aceitáveis para determinações de distribuição de agregados conforme KEMPER (1965), KIEHL (1979) e ZALAMENA et al. (2007).

TABELA 3 - ESTATÍSTICAS REFERENTES À AGREGAÇÃO DO SOLO, CONSIDERANDO CINCO CLASSES DE AGREGADOS (TAMANHO EM mm) E QUATRO REPETIÇÕES PARA O LVdq DA MICROBACIA DO CÓRREGO DO ESPRAIADO - RIBEIRÃO PRETO (SP)

\begin{tabular}{|c|c|c|c|c|c|c|}
\hline Solo & Estatística & $\begin{array}{c}\text { Agregado } 1 \\
(>2 \mathrm{~mm})\end{array}$ & $\begin{array}{c}\text { Agregado } 2 \\
(2-1 \mathrm{~mm})\end{array}$ & $\begin{array}{c}\text { Agregado } 3 \\
(1-0,5 \mathrm{~mm})\end{array}$ & $\begin{array}{c}\text { Agregado } 4 \\
(0,5-0,25 \mathrm{~mm})\end{array}$ & $\begin{array}{l}\text { Agregado } 5 \\
(<0,25 \mathrm{~mm})\end{array}$ \\
\hline \multirow{4}{*}{ LVdq } & Média & 8,3 & 39,9 & 15,5 & 10,3 & 26,0 \\
\hline & Mediana & 8,3 & 39,9 & 15,5 & 10,3 & 26,0 \\
\hline & $\begin{array}{l}\text { Coeficiente de } \\
\text { Variação }\end{array}$ & 8,2 & 5,5 & 7,8 & 9,4 & 13,3 \\
\hline & Intervalo & - & 4,8 & 2,9 & 1,9 & 8,3 \\
\hline
\end{tabular}




\section{TABELA 4 - ESTATÍSTICAS REFERENTES À AGREGAÇÃO DO SOLO, CONSIDERANDO CINCO CLASSES DE AGREGADOS (TAMANHO EM mm) E QUATRO REPETIÇÕES PARA O RQO DA MICROBACIA DO CÓRREGO DO ESPRAIADO - RIBEIRÃO PRETO (SP)}

\begin{tabular}{c|l|c|c|c|c|c}
\hline Solo & Estatística & $\begin{array}{c}\text { Agregado 1 } \\
(>2 \mathrm{~mm})\end{array}$ & $\begin{array}{c}\text { Agregado 2 } \\
(2-1 \mathrm{~mm})\end{array}$ & $\begin{array}{c}\text { Agregado 3 } \\
(1-0,5 \mathrm{~mm})\end{array}$ & $\begin{array}{c}\text { Agregado 4 } \\
(0,5-0,25 \mathrm{~mm})\end{array}$ & $\begin{array}{c}\text { Agregado 5 } \\
(<0,25 \mathrm{~mm})\end{array}$ \\
\hline \multirow{4}{*}{ RQo } & Média & 2,4 & 5,8 & 15,1 & 65,0 & 11,9 \\
\cline { 2 - 7 } & Mediana & 2,4 & 5,8 & 15,7 & 64,9 & 11,8 \\
\cline { 2 - 7 } & $\begin{array}{l}\text { Coeficiente de } \\
\text { Variação }\end{array}$ & 10,8 & 3,6 & 18,0 & 3,1 & 8,2 \\
\cline { 2 - 7 } & Intervalo & 0,6 & 0,5 & 5,9 & 4,8 & 2,2 \\
\hline
\end{tabular}

\section{FIGURA 1- ANÁLISE DE AGREGADO DOS SOLOS LVdq E RQo}

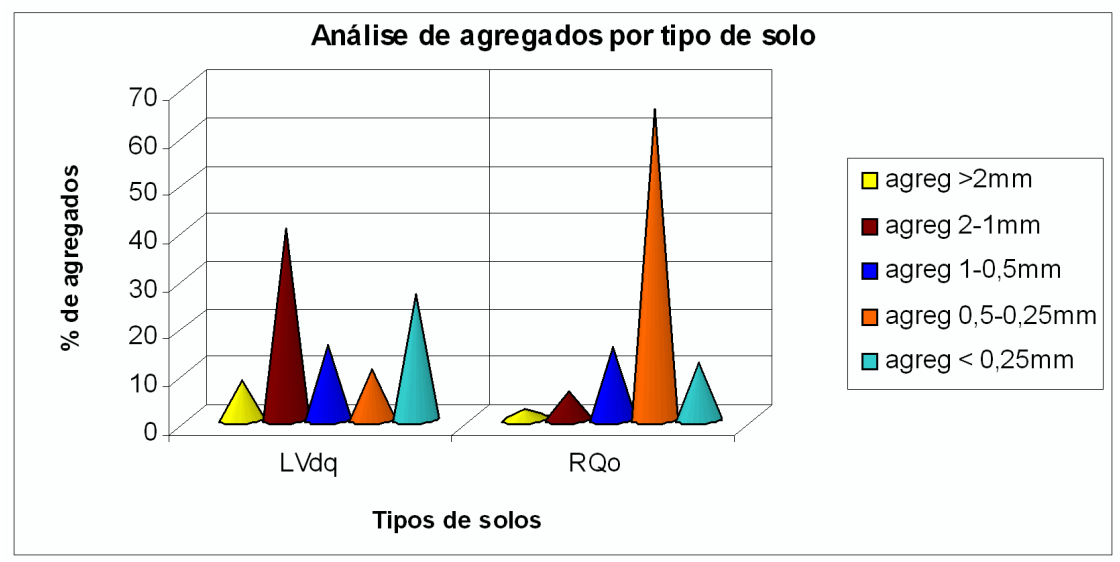

$\mathrm{Na}$ análise de condutividade hidráulica do solo saturado (Ksat), durante sete horas, observou-se variação nos valores de ano para ano nos dois tipos de solo estudados (Tabela 5). Essa variação pode ser atribuída aos distintos pontos de análise tomados a cada ano, bem como ao fato de que a condutividade hidráulica do mesmo solo pode variar em decorrência dos processos químicos, físicos e biológicos que ocorrem continuamente. Observou-se também, em todos os anos, que houve semelhança na intensidade de infiltração da água para os dois tipos de solos. Ambos acusaram alta taxa de infiltração na primeira hora, com decréscimo paulatino nas horas subsequentes. Ao mesmo tempo, os valores de infiltração ao longo do período analisado (7h) foram sempre maiores para o solo RQo. Tal fato pode ser explicado pelo seu elevado percentual de areia e, presumivelmente, pela alta macroporosidade que propiciam maior condutividade hidráulica.

Comparando a condutividade hidráulica dos dois solos, tanto o LVdq quanto RQo apresentaram elevadas taxas de infiltração. Esses resultados são coerentes quando se analisa a Tabela 6, caracterizando a influência da textura e da estrutura na condutividade hidráulica do solo.

No RQo, as partículas da fração areia (grossa + fina) com valores médios entre 87-92\% apresentaram alto grau de arredondamento, propiciando a formação de grande 
quantidade de espaços vazios, principalmente macroporos. Essa condição de arredondamento foi favorecida pela gênese fluvio-eólica, decorrente da deposição do material sedimentar que contribuiu para a formação do Aquífero Guarani. Apesar de esse solo apresentar estrutura maciça, verificou-se a dominância de $65 \%$ de agregados estáveis na classe 0,5-0,25 mm de diâmetro, os quais funcionam como partículas mais grosseiras, com espaços porosos maiores, favorecendo as taxas mais altas de condutividade hidráulica.

No LVdq, a presença de areia (grossa + fina) em quantidade relativamente elevada (cerca de 71 a 76\%) confere textura do tipo franco-arenosa. Essa textura, aliada à estrutura granular, com cerca de $40 \%$ de agregados na classe de 2,0-1,0 mm de diâmetro contribui para taxa relativamente elevada de Ksat, em comparação ao RQo.

\section{TABELA 5 - CONDUTIVIDADE HIDRÁULICA DO SOLO SATURADO (KSAT) OBTIDA PARA O LVDQ - LATOSSOLO VERMELHO DISTRÓFICO PSAMÍTICO E RQO - NEOSSOLO QUARTZARÊNICO ÓRTICO NA PROFUNDIDADE $0-20 \mathrm{~cm}$, DURANTE OS PERÍODOS DE 1996, 1998, 2000, 2001 E 2003}

\begin{tabular}{|c|c|c|c|c|c|c|c|c|c|c|c|c|c|c|}
\hline \multirow[t]{3}{*}{ Data } & \multicolumn{7}{|c|}{ LVdq } & \multicolumn{7}{|c|}{ RQO } \\
\hline & \multicolumn{14}{|c|}{$\mathrm{cm} \cdot \mathrm{h}^{-1}$} \\
\hline & $1^{\text {a hora }}$ & $2^{\mathrm{a}}$ hora & $3^{a}$ hora & $4^{a}$ hora & $5^{a}$ hora & $6^{a}$ hora & $7^{a}$ hora & $1^{\mathrm{a}}$ hora & $2^{a}$ hora & $3^{a}$ hora & $4^{a}$ hora & $5^{a}$ hora & $6^{a}$ hora & $7^{\mathrm{a}}$ hora \\
\hline $1996 \underline{\underline{1}}$ & 49,36 & 42,10 & 38,73 & 35,95 & 32,18 & 30,24 & 29,27 & 53,22 & 50,12 & 48,28 & 46,43 & 45,19 & 45,07 & 43,13 \\
\hline $1998 \underline{\underline{y}}$ & 43,25 & 40,51 & 40,18 & 37,55 & 37,04 & 34,08 & 32,11 & 50,43 & 49,22 & 47,58 & 46,05 & 44,64 & 43,19 & 40,15 \\
\hline $2000 \underline{\underline{\mu}}$ & 45,42 & 44,13 & 43,71 & 40,86 & 36,07 & 30,14 & 30,02 & 55,30 & 53,90 & 50,27 & 48,87 & 46,35 & 45,22 & 39,97 \\
\hline $2001 \underline{\underline{2}}$ & 47,17 & 46,33 & 45,02 & 41,48 & 35,75 & 31,49 & 28,66 & 53,88 & 51,31 & 50,17 & 47,65 & 45,37 & 43,85 & 41,03 \\
\hline $2003 \underline{3}$ & 51,34 & 47,15 & 46,53 & 40,74 & 38,63 & 34,05 & 31,50 & 56,83 & 54,46 & 53,92 & 49,04 & 48,06 & 47,73 & 42,52 \\
\hline Média & 47,31 & 44,04 & 42,83 & 39,32 & 35,93 & 32,00 & 30,19 & 53,93 & 51,80 & 50,04 & 47,61 & 45,92 & 45,01 & 41,36 \\
\hline
\end{tabular}

Fonte: 1/EMBRAPA, 1998; 2/EMBRAPA, 2001; 3/EMBRAPA, 2004.

\section{TABELA 6 - CONDUTIVIDADE HIDRÁULICA SATURADA (KSAT), TEXTURA E ESTRUTURA DOS SOLOS LVDQ E RQO DAS ÁREAS DE RECARGA DO AQUÍFERO GUARANI, MICROBACIA DO CÓRREGO ESPRAIADO, RIBEIRÃO PRETO (SP)}

\begin{tabular}{c|c|c|c}
\hline $\begin{array}{c}\text { Classe de } \\
\text { Solo }\end{array}$ & $\begin{array}{c}\text { Textura } \\
\text { Predominante }^{\mathbf{1}}\end{array}$ & $\begin{array}{c}\text { Estrutura } \\
\text { Predominante }^{\mathbf{1}}\end{array}$ & $\begin{array}{c}\text { Condutividade } \\
\text { Hidráulica }^{\mathbf{2}}\end{array}$ \\
\hline $\begin{array}{c}\text { Latossolo Vermelho Distrófico psamítico } \\
\text { (LVdq) }\end{array}$ & Franco-arenosa & Granular & Média/Alta \\
\hline $\begin{array}{c}\text { Neossolo Quartzarênico } \\
\text { Órtico (RQo) }\end{array}$ & Areia & $\begin{array}{c}\text { Maciça (com } \\
\text { comportamento } \\
\text { granular) }\end{array}$ & Alta/Muito Alta \\
\hline
\end{tabular}

Fonte: 1/SANTOS et al. (2005); 2/GOMES et al. (2002); 2/MIKLÓS e GOMES (1996). 


\section{CONCLUSÃO}

A partir dos resultados e discussões realizadas foi possível concluir que os solos Latossolo Vermelho Distrófico psamítico (LVdq) e Neossolo Quartzarênico Órtico (RQo) apresentaram elevada condutividade hidráulica saturada (Ksat), com valores que variam de 40 a $55 \mathrm{~cm} . \mathrm{h}^{-1}$. Todavia, verificou-se que no RQo esses valores foram ainda mais elevados devido à dominância das partículas na fração areia (87 a 92\%), com alto grau de arredondamento e à predominância (65\%) de agregados estáveis na classe 0,5 -0,25 mm de diâmetro. Os valores relativamente altos de Ksat para o LVdq se devem à presença de partículas na fração areia (71-77\%), com alto grau de arredondamento e à predominância (40\%) de agregados na classe 2-1 mm de diâmetro. Os valores de Ksat dos dois solos estudados indicam situação de alta vulnerabilidade natural para as áreas de recarga do Aquífero Guarani, expondo-as a uma situação de risco de contaminação do lençol freático/ profundo, frente aos agroquímicos comumente usados nas atividades agrícolas da região.

\section{ABSTRACT \\ HYDRAULIC CONDUCTIVITY EVALUATION IN RECHARGE AREAS OF THE GUARANI AQUIFER - ESPRAIADO WATERSHED, SP (BRAZIL)}

The hydraulic conductivity of the saturated soil (Ksat) is a great relevance parameter in the evaluation studies of the natural vulnerability and therefore the environmental risk assessment. When it comes to fragile areas, as they are the recharge areas of sedimentary aquifers, the hydraulic conductivity shows even more important. For this study was adopted a representative area of the direct recharge of the Guarani aquifer. It is characterized by the Espraiado watershed, located in Ribeirão Preto district, SP (Brasil). The main soils of this area are: Quatzipsammentic Haplorthox (QH) and Typic Quartzipsamment (TQ). For the hydraulic conductivity (Ksat) of these soils, was used the saturated column method, whose values were correlated with those of granulometry or particle size and soil structure, considered as parameters more directly linked to Ksat. The direct correlations were between Ksat values and those sandy fraction. For the structure, this correlation occurred indirectly, indicating lower influence of this parameter on the hydraulic conductivity.

KEY-WORDS: WATER CONTAMINATION; NATURAL VULNERABILITY; FRAGILE AREAS; ENVIRONMENTAL QUALITY.

\section{REFERÊNCIAS}

1 BRADY, N. C. Natureza e propriedade do solo. 6. ed. Rio de janeiro: Freitas, 1983. 647 p.

2 EMBRAPA. Centro Nacional de Pesquisa de Solos. Manual de métodos de análise de solo. Rio de Janeiro, 1979. 212 p. (EMBRAPA - CNPS. Documentos, 1).

3 EMBRAPA. Centro Nacional de Pesquisa de Monitoramento e Avaliação de Impacto Ambiental. Impacto ambiental e implicações socioeconômicas da agricultura intensiva em água subterrânea. Jaguariúna: Embrapa Meio Ambiente, 1998. 26 p (Relatório final de projeto).

4 EMBRAPA. Centro Nacional de Pesquisa de Solos. Sistema brasileiro de classificação de solos. Brasília: Embrapa Produção de Informação; Rio de Janeiro: Embrapa Solos, 1999. 412 p.

5 EMBRAPA. Centro Nacional de Pesquisa de Monitoramento e Avaliação de Impacto Ambiental. Uso agrícola das áreas de afloramento do Aquífero Guarani e implicações na qualidade da água subterrânea. Jaguariúna: Embrapa Meio Ambiente, 2001. 32 p. (Relatório final de projeto).

6 EMBRAPA. Centro Nacional de Pesquisa de Monitoramento e Avaliação de Impacto Ambiental. Uso agrícola atual e avaliação qualitativa dos recursos hídricos das principais áreas críticas localizadas nas porções de afloramento do Aquífero Guarani em território brasileiro. Jaguariúna: Embrapa Meio Ambiente, 2004. 12 p. (Relatório final de projeto). 
7 FERNANDES, B.; GALLOWAY, H. M.; BRONSON, R. D.; MANNERING, J. V. Condutividade hidráulica do solo saturado em três sistemas de manejo. Rev. Ceres, v. 30, p. 232-241, 1983.

8 GOMES, M. A. F.; SPADOTTO, C. A.; PESSOA, M. C. P. Y. Avaliação da vulnerabilidade natural do solo em áreas agrícolas: subsídio à avaliação do risco de contaminação do lençol freático por agroquímicos. Pesticidas: r. ecotoxicol. e meio ambiente, v. 12, p. 169 -179, 2002.

9 KEMPER, W. D. Aggregate stability. In: BLACK, C. A. (ed.). Methods of soil analysis. Madison: American Society of Agronomy, 1965. p. 511-519.

10 KHIEL, E. J. Manual de edafologia: relações solo-planta. São Paulo: Ed. Agronômica Ceres, 1979. $262 \mathrm{p}$.

11 KRAMER, P. J. 1969. Plant and soil water relationships: a modern synthesis. New York: McGraw-Hill Book,1969. 482 p.

12 LACERDA, N. B.; ZERO, V. M.; BARILLI, J.; MORAES, M. H.; BICUDO, S. J. Efeito de sistemas de manejo na estabilidade de agregados de um Nitossolo Vermelho. Eng. Agríc., Jaboticabal, v. 25, n. 3, p. 686695, set./dez. 2005.

13 MIKLÓS, A. A. W.; GOMES, M. A. F. Levantamento semi-detalhado dos solos da Bacia Hidrográfica do Córrego Espraiado, Ribeirão Preto-SP. Jaguariúna, 1996. 48 p. (Relatório de Consultoria).

14 SANTOS, R. D.; LEMOS, R. C.; SANTOS. H. G.; KER, J. C.; ANJOS, L. H. C. Manual de descrição e coleta de solo no campo. 5. ed. Viçosa: SBCS, 2005. 100 p.

15 SÃO PAULO. Instituto Geológico. Mapeamento da vulnerabilidade e risco de poluição das águas subterrâneas no Estado de São Paulo. São Paulo: CETESB/DAEE, 1997. 144 p. 2 v. (Série Documentos).

16 YOUNGS, E. G. Hydraulic conductivity of saturated soils. In: SMITH, K. A.; MULLINS, C. E. (Eds.). Soil analysis: physical methods. New York: M. Dekker, 1991. p. 161-207.

17 ZALAMENA, J.; DALMOLIN, R. S. D.; MIGUEL, P.; MEDEIROS, P. S. C.; FINK, J.; DOTTO, A. C.; COUTO, R. M. R.; DENEGA, G. L. Distribuição de agregados em um Argissolo Vermelho sob diferentes usos. In: CONGRESSO BRASILEIRO DE CIÊNCIA DO SOLO, 31., Gramado (RS), 2007. Anais... Gramado: SBCS, 2007. p. 4. 